\title{
Window-coupled nanolayers: window shape influence on one-particle and two-particle eigenstates
}

\author{
A. S. Bagmutov, I. Y. Popov \\ ITMO University, Kronverkskiy, 49, Saint Petersburg, 197101, Russia \\ bagmutov94@mail.ru,popov1955@gmail.com
}

DOI 10.17586/2220-8054-2020-11-6-636-641

\begin{abstract}
In this work, we present a number of numerical results for the bound state energies of one and two-particle systems in two adjacent 3D layers, connected through a window. We investigate the relation between the shape of a window and energy levels, as well as number of eigenfunction's nodal domains.
\end{abstract}

Keywords: nanolayer, eigenfunction, Hartree-Fock model.

Received: 13 September 2020

Revised: 1 November 2020

\section{Introduction}

In general, the waveguides can be modelled by the Dirichlet Laplacians in infinite planar strips and multidimensional layers, the spectra of which is a focus of many works in past decades. The problem is trivial as long as the strip or layer is straight, because one can then employ separation of variables. However, already a local perturbation such as bending, deformation, or a change of boundary conditions can produce a non-empty discrete spectrum. As examples of possible perturbations, we indicate local deformation of the boundary condition [1,2], bending [3-6] or twisting [7,8] the waveguide. Perturbation by adding a potential is considered in [9], and by a magnetic field in [10,11], or by a second order differential operator as in [12]. The type of systems we are interested in is two adjacent parallel waveguides, coupled by the windows cut out in a common boundary. The two-dimensional case was studied quite intensively, we refer here to [13-20] (see also references therein). It was shown that the perturbation by the window(s) is a negative one, i.e., it leads to the presence of the isolated bound states below the essential spectrum; the latter is invariant w.r.t. to the window(s). In the case of one window, it was shown in $[13,15,17]$ that widening the window one produces more and more isolated eigenvalues. They appear when the windows length passes through certain critical values; this phenomenon was studied in detail and the asymptotics expansions for the emerging eigenvalues were obtained, see $[13,15,19]$. In the three-dimensional case of window-coupled layers, it was shown that a small window generates one simple isolated eigenvalue emerging from the threshold of the essential spectrum [18]. This work also contains two-sided asymptotic estimates for the eigenvalue. The asymptotic expansion for this eigenvalue has been constructed formally in [21]. In the present work, we present a number of numerical results for two adjacent layers in $\mathbb{R}^{3}$, coupled through holes in the common boundary. Such a system was studied in [22], where it was shown that the window produces eigenvalues emerging from the threshold of essential spectrum, as the window passes through certain critical shapes. We continuously change window shape and look at evolution of bound states with fixed number and position of nodal domains. The questions concerning to number and position of nodal domains for the Dirichlet Laplacians are a point of active research. The first step was Courant's nodal theorem, and since then, various different cases have been investigated, such as nodal domains for quantum graphs ( $[29,30])$ and in a sphere ( [31]). Also, within this geometry, we consider a case of two particles, with different shapes of a window. We make numerical calculations, with the application of Hartree-Fock estimation method (for the Hartree approximation accuracy, see [28]). As for previous studies of the multi-particle problems in deformed waveguides, see, e.g., [23-27]. We are concerned with the following two questions: how the energies of bound states and number/position of nodal domains are dependent on shape of a window and what is the relation between one and two-particle cases.

\section{Hartree-Fock approach}

Let us describe the Hartree-Fock model and algorithm. 
We start with the Hamiltonian

$$
\hat{H}=\sum_{k}\left(-\Delta_{k}+U_{k}\right)+\frac{1}{2} \sum_{\substack{j, k \\ j \neq k}} V_{j k}=\sum_{k} \hat{H}_{k}+\frac{1}{2} \sum_{\substack{j, k \\ j \neq k}} V_{j k},
$$

where $\Delta_{k}=\frac{\partial^{2}}{\partial x_{k}{ }^{2}}$ is the Laplace operators, acting on coordinates $x_{k}$ of the $k$-th particle, $U_{k}$ is the potential of the external field, $V_{j, k}$ is the potential of particles interaction, $\hat{H}_{k}=\left(-\Delta_{k}+U_{k}\right)$. Then, we should approximate many-particle wave function by the Slater determinant:

$$
\Psi\left(x_{1}, x_{2}, \ldots, x_{n}\right)=\frac{1}{\sqrt{n !}}\left|\begin{array}{ccc}
\psi_{1}\left(x_{1}\right) & \ldots & \psi_{n}\left(x_{1}\right) \\
\ldots & \ldots & \ldots \\
\psi_{1}\left(x_{n}\right) & \ldots & \psi_{n}\left(x_{n}\right)
\end{array}\right|
$$

where $\psi_{k}$ are single-particle wave functions and $x_{k}=\left(r_{k}, s_{k}\right)$, where $r_{k}$ and $s_{k}$ are the spatial and spin coordinates of the $k$-th particle. Following the Hartree-Fock method, we insert (2) in (1) and use variation of energy functional $\langle\Psi \mid \hat{H} \Psi\rangle$ to get the Hartree-Fock equations:

$$
\begin{array}{r}
{\left[\hat{H}_{k}+\sum_{j, j \neq k} \int \psi_{j}^{*}\left(x_{j}\right) V_{j k} \psi_{j}\left(x_{j}\right) d x_{j}\right] \psi_{k}\left(x_{k}\right)-} \\
-\sum_{j, j \neq k}\left(\int \psi_{j}^{*}\left(x_{j}\right) V_{j k} \psi_{k}\left(x_{j}\right) d x_{j}\right) \psi_{j}\left(x_{k}\right)=E_{k} \psi_{k}\left(x_{k}\right) .
\end{array}
$$

Then, we should consider the spin of the particles. We ignore the spin-orbit interaction. Let $n^{\uparrow}$ be the number of particles with spin 0.5 and $n^{\downarrow}$ - particles with spin -0.5 . The wave functions of these particles are $\psi_{k}^{\uparrow}$ and $\psi_{k}^{\downarrow}$. Then, due to independence of $V_{j k}$ from the spin variable, one has

$$
\int \psi_{j}^{*}\left(x_{j}\right) V_{j k} \psi_{k}\left(x_{j}\right) d x_{j}=\delta_{s_{j} s_{k}} \int \psi_{j}^{* s_{j}}\left(r_{j}\right) V_{j k} \psi_{k}^{s_{k}}\left(r_{j}\right) d r_{j}
$$

where $\delta_{s_{j} s_{k}}$ is the Kronecker symbol, indices $s_{j}, s_{k}$ take values $\uparrow, \downarrow$.

As an interaction potential, we use the delta-potential: $V_{j k}=U \delta\left(r_{j}-r_{k}\right)$, where $U$ is a constant. Using the deltapotential and (4), from (3) we get the following system:

$$
\begin{aligned}
& H_{k} \psi_{j}^{\uparrow}\left(r_{k}\right)+U \sum_{j}^{n \downarrow}\left(\left|\psi_{j}^{\downarrow}\left(r_{k}\right)\right|^{2} \psi_{k}^{\uparrow}\left(r_{k}\right)\right)=E_{k}^{\uparrow} \psi_{k}^{\uparrow}\left(r_{k}\right), \quad\left(k=1, . ., n^{\uparrow}\right), \\
& H_{k} \psi_{j}^{\downarrow}\left(r_{k}\right)+U \sum_{j}^{n \uparrow}\left(\left|\psi_{j}^{\uparrow}\left(r_{k}\right)\right|^{2} \psi_{k}^{\downarrow}\left(r_{k}\right)\right)=E_{k}^{\downarrow} \psi_{k}^{\downarrow}\left(r_{k}\right), \quad\left(k=1, . ., n^{\downarrow}\right) .
\end{aligned}
$$

To solve the system, we used the following iterative algorithm:

(1) Find one-particle stationary solutions for a case of potential $U_{k}=F z_{k}$ and arbitrarily choose $N$ solutions as initial approximations for $\psi_{i}\left(x_{i}\right)$.

(2) For each particle, calculate a potential $P_{i}=U \sum_{j}\left|\psi_{j}\left(x_{j}\right)\right|^{2}$, where sum includes particles with the opposite spins, then, use it to calculate a set of solutions.

(3) From each of $N$ sets choose a bound solution. (Here we can use arbitrary additional criteria, to speed up the process.)

(4) Check if new solutions are close enough to previous ones. If not, return to step 2.

\section{Results}

The system we are studying consists of two adjacent infinite 3D layers of constant width, with a common border. In the common border there is a window, connecting two layers. We consider bound states for systems of one and two particles with delta interaction. On the borders of layers, we assume the Dirichlet boundary condition. The main focus of the study is the relation between shape of a window and energies of bound states. We consider two types of windows: elliptic windows and windows shaped like Cassini ovals (peanut-shaped), which transform into two separate circular windows. We show the dependence of bound state energy $E$ as a function of parameter $d$, which in the case 

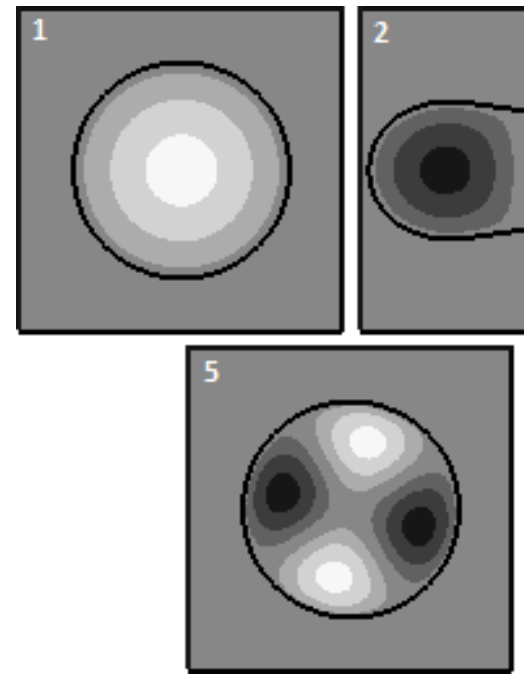
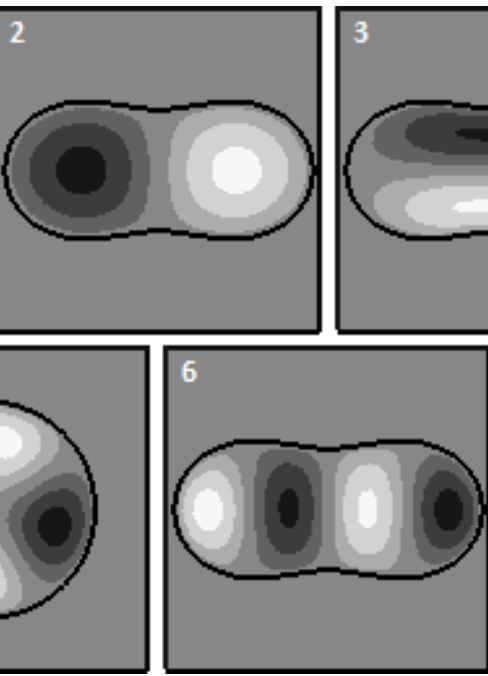
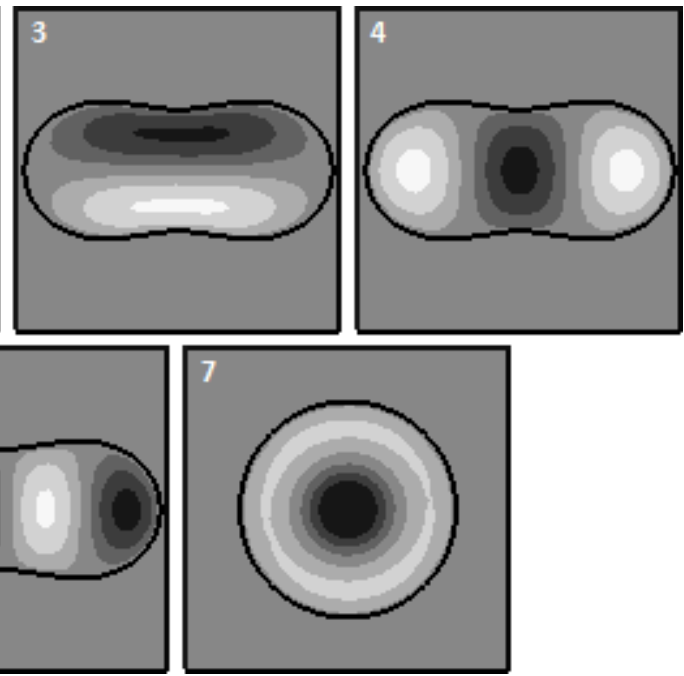

FIG. 1. Examples of bound state types, as a 2D slice along window's plane. Labels: 1 - one, 2 two, 3 - transversal two, 4 - three, 5 - square four, 6 - row four, 7 - ring.

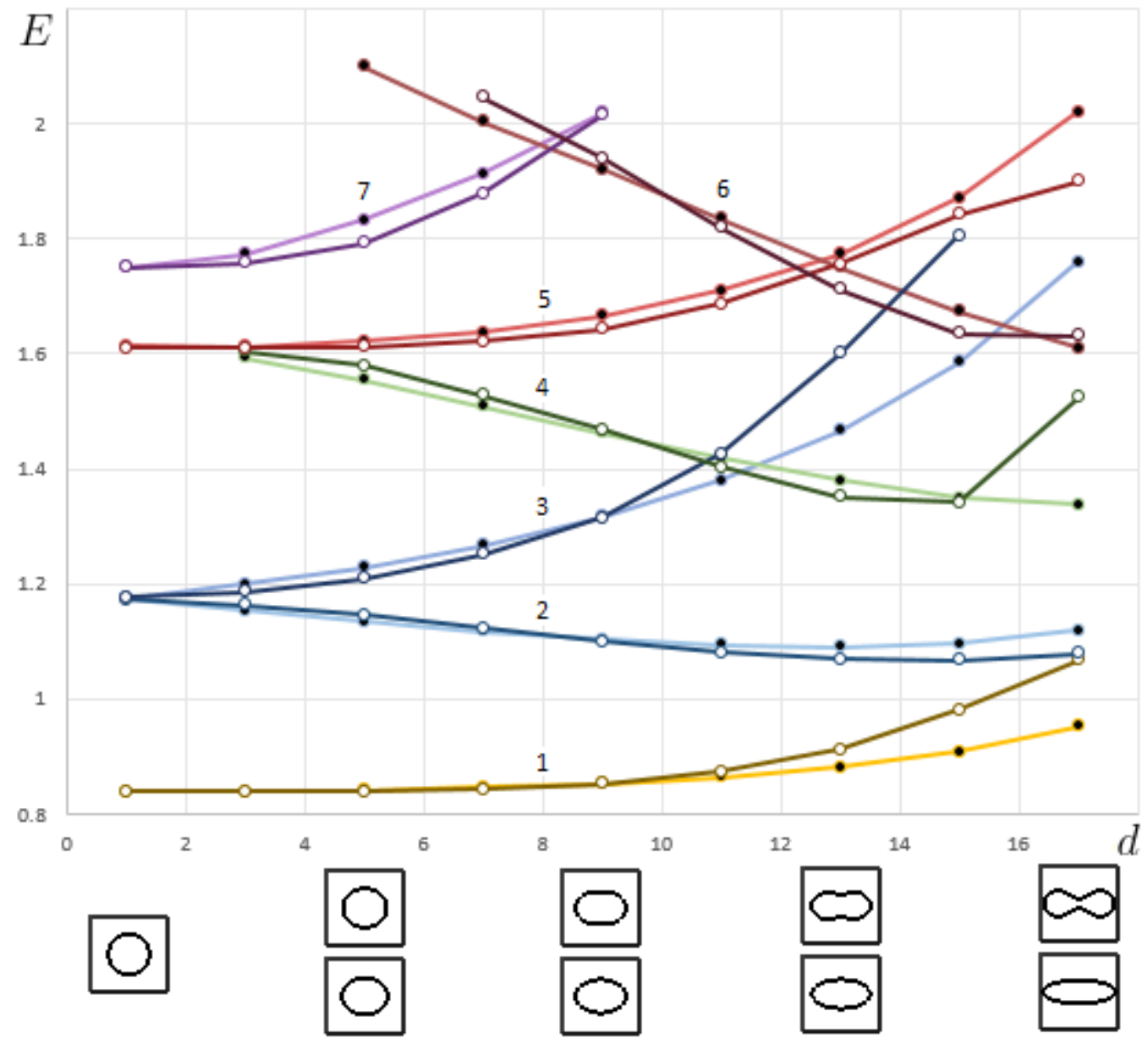

FIG. 2. Energies of different one-particle types of states as functions of distance between foci of Cassini ovals. The faint lines represent energies of the same type for the elliptic window of the same width. All windows have the same area. The types are labeled according to the enumeration in Fig. 1. Shapes of the windows are shown below the $d$ axis. 
of Cassini ovals, represents a distance between oval foci and for two apertures it is a distance between their centers. Ellipses are chosen to match Cassini ovals' width. All windows have the same area.

First, we consider the case of one particle. If we examine 2D slice of eigenfunctions through the plane of a window, we can distinguish a number of persistent types of solutions, categorized by the number and position of nodal domains. The Courant's nodal theorem states that for the Dirichlet Laplacian, the number of nodal domains can not exceed the index of a state in a list of all states, sorted by eigenvalues. In our case, we will first follow seven types, which are shown in Fig. 1. These types of bound states show consistent behavior with change of parameter $d$ and their energies change in different ways, according to the geometry of the type. Results for the cases of an elliptic and Cassini oval shaped windows are shown on the Fig. 2.

As we can see, for the most types, bound levels tend in the positive direction with the increasing deformation of circular window, but for certain types, it is reversed. Let us note some features. The first two states For Cassini ovals converge to the same energy, because the first state evolve in a copy of the second, but with both peaks pointing in the same direction. The transversal two (3) and the three (4) types are affected by the final Cassini evolution most of all (the energy of the former for the last aperture is too high for the shown range), because their constant-sign zone is in the center and deformed by the closing gap. With the further closing of a bridge, type (4) will get closer to type (6), but in conformity with the Courant's theorem, will not exceed it.

For the two-particle system, we consider the Cassini oval window, and look at the lowest bound energy level for the different strengths of delta interaction (see Fig. 3), and compare it to the first two levels from the Fig. 2. We also extended the deformation of the window, adding three states with two circular windows at the end. For the case of two holes, all different strengths have indistinguishable on our scale levels. The plot shows that with the increase of interaction strength two particle states tend from first, toward second type of state. The energy for two holes is almost independent on the distance between the holes.

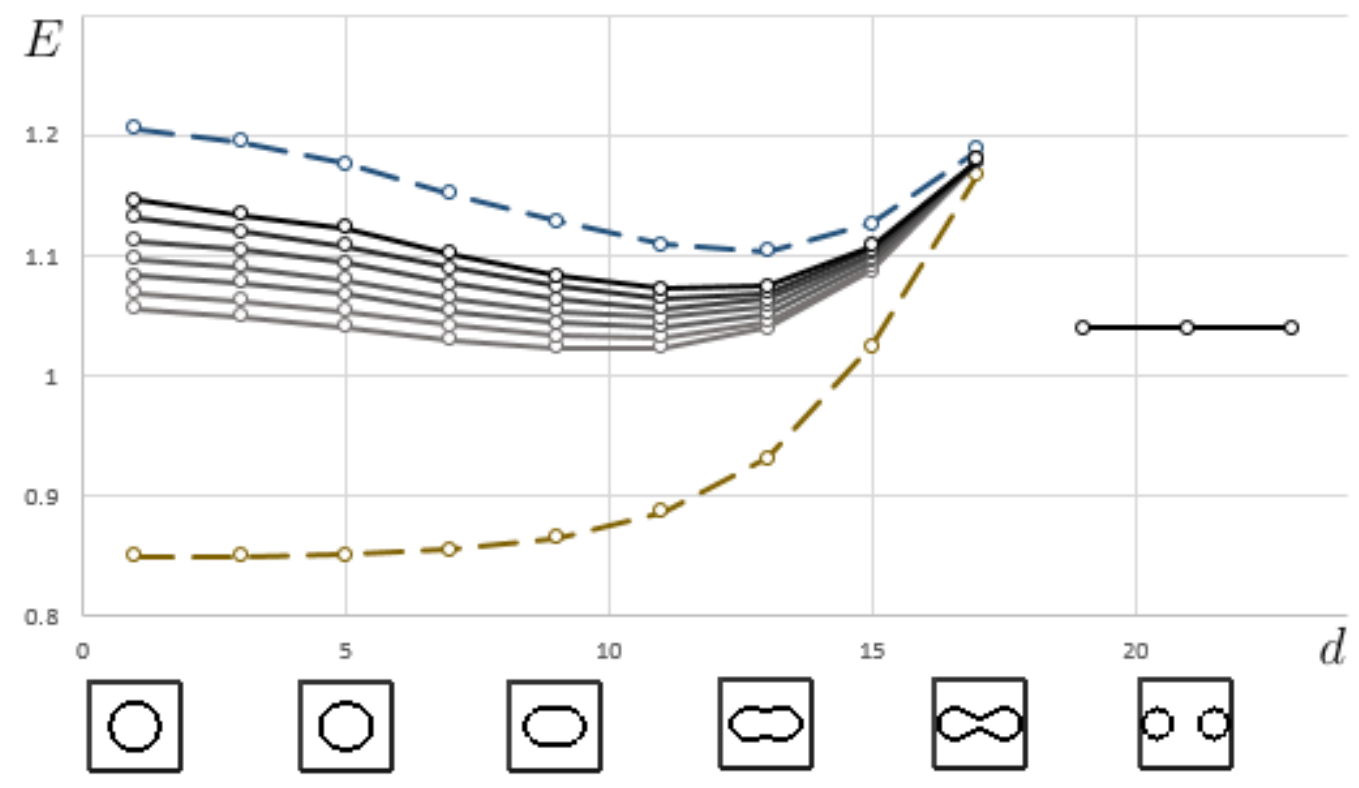

FIG. 3. Lowest bound energies for two particles. Dotted plots represent the first two one-particle types for Cassini Windows from Fig. 2 for comparison, which differ because of different precision for the case of more complex computation. Grey lines represent energies of each particle in lowest two-particle bound states, each line corresponds to different strength of delta-interaction. The strengths used: $30,50,85,140,250,500,1000$ (arbitrary units) from the lowest to the highest line respectively. For the case of two separate circular openings all strengths have very similar energies, plotted in the right part.

On the Fig. 4 we compare the energies of elliptic bound state with the Cassini ones for the same interaction strength. The plots for different interaction strengths show the same relations. 


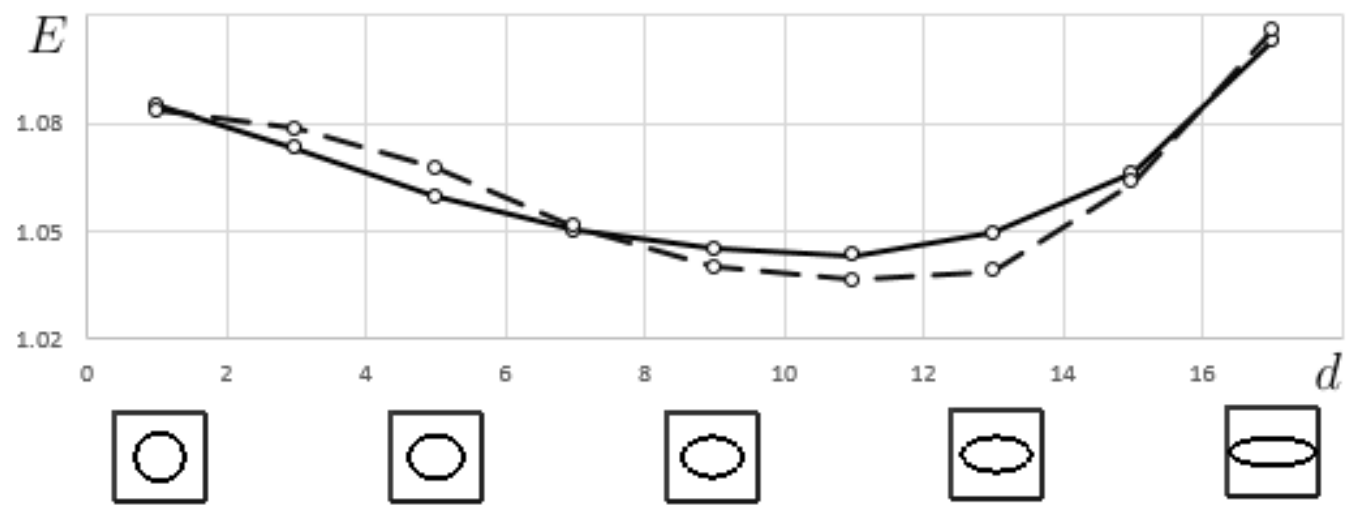

FIG. 4. Comparison of two particle bound state energies for elliptic window (solid line), with corresponding levels for Cassini oval window (dotted line).

\section{Acknowledgements}

This work was partially financially supported by grant 20-31-90050 of Russian Foundation for Basic Research.

\section{References}

[1] Borisov D., Exner P., Gadylshin R., Krejcirik D. Bound states in weakly deformed strips and layers, Annales Henri Poincare, 2001, 2(3), P. 553-572.

[2] Bulla W. and Renger W. Existence of bound states in quantum waveguides under weak conditions. Lett. Math. Phys., 1995, 35(1), P. 1-12.

[3] Duclos P. and Exner P. Curvature-induced bound state in quantum waveguides in two and three dimensions. Rev. Math. Phys., 1995, 7(1), P. 73-102.

[4] Duclos P., Exner P., Stovicek P. Curvature-induced resonances in a two-dimensional Dirichlet tube. Annales Henri Poincare, 1995, 62(1), P. 81-101.

[5] Chenaud B., Duclos P., Freitas P. and Krejcirik D. Geometrically induced discrete spectrum in curved tubes. Diff. Geom. Appl., 2005, 23(2), P. 95-105.

[6] Exner P. and Vugalter S.A. Bound States in a Locally Deformed Waveguide: The Critical Case. Lett. Math. Phys., 1997, 39(1), P. 59-68.

[7] Briet Ph., Kovarik H., Raikov G. and Soccorsi E. Eigenvalue asymptotics in a twisted waveguide. Comm. PDE, 2009, 34(8), P. 818-836.

[8] Ekholm T., Kovarik H. and Krejcirik D. A Hardy inequality in twisted waveguides. Arch. Rat. Mech. Anal., 2008, 188(2), P. $245-264$.

[9] Duclos P. and Exner P. Curvature-induced bound state in quantum waveguides in two and three dimensions. Rev. Math. Phys., 1995, 7(1), P. 73-102.

[10] Borisov D., Ekholm T. and Kovarik H. Spectrum of the magnetic Schrodinger operator in a waveguide with combined boundary conditions. Annales Henri Poincare, 2005, 6(2), P. 327-342.

[11] Ekholm T. and Kovarik H. Stability of the magnetic Schrodinger operator in a waveguide. Comm. PDE, 2005, 30(4), P. 539-565.

[12] Grushin V.V. On the eigenvalues of finitely perturbed laplace operators in infinite cylindrical domains. Math. Notes, 2004, 75(3), P. 331-340.

[13] Borisov D. Discrete spectrum of a pair of non-symmetric waveguides coupled by a window. Sbornik Mathematics, 2006, 197(4), P. 475-504.

[14] Borisov D. and Exner P. Distant perturbation asymptotics in window-coupled waveguides. I. The non-threshold case. J. Math. Phys., 2006, 47(11), P. 113502-(1-24).

[15] Borisov D., Exner P. and Gadylshin R. Geometric coupling thresholds in a two-dimensional strip. Journal of Mathematical Physics, 2002, 43(12), P. 6265-6278.

[16] Bulla W., Gesztesy F., Renger W., Simon B. Weakly coupled bound states in quantum waveguides. Proc. Amer. Math. Soc., 1997, 125(5), P. $1487-1495$

[17] Exner P., Seba P., Tater M., and Van?ek D. Bound states and scattering in quantum waveguides coupled laterally through a boundary window. J. Math. Phys., 1996, 37(10), P. 4867-4887.

[18] Exner P. and Vugalter S. Bound-state asymptotic estimate for windowcoupled Dirichlet strips and layers. J. Phys. A., 1997, 30(22), P. 78637878.

[19] Gadylshin R. On regular and singular perturbation of acoustic and quantum waveguides. Comptes Rendus Mechanique, 2004, 332(8), P. 647652.

[20] Vorobiev A.M., Bagmutov A.S., Popov A.I. On formal asymptotic expansion of resonance for quantum waveguide with perforated semitransparent barrier. Nanosystems: Physics, Chemistry, Mathematics, 2019, 10(4), P. 415-419.

[21] Popov I.Yu. Asymptotics of bound states and bands for laterally coupled waveguides and layers. J. Math. Phys., 2002, 43(1), P. 215-234.

[22] Borisov D. On the spectrum of two quantum layers coupled by a window. J. Phys. A: Math. Theor., 2007, 40(19), P. 5045-5066.

[23] Linde H. Geometrically induced two-particle binding in a waveguide. J. Phys. A: Math. Gen., 2006, 39(18), P. 5105-5114.

[24] Popov S.I., Gavrilov M.I. and Popov I.Yu., Two interacting particles in deformed nanolayer: discrete spectrum and particle storage. Phys. Scripta, 2012, 86(3), P. 035003.

[25] Melikhov I.F. and Popov I.Yu. Hartree-Fock approximation for the problem of particle storage in deformed nanolayer. Nanosystems: Physics, Chemistry, Mathematics, 2013, 4(4), P. 559-563. 
[26] Popov I.Y., Bagmutov A.S., Melikhov I.F., Najar H. Numerical analysis of multi-particle states in coupled nano-layers in electric field. AIP Conference Proceedings, 2020, 2293, P. 360006.

[27] Popov I.Y., Melikhov I.F. Multi-particle bound states in window-coupled 2D quantum waveguides. Chinese J. of Physics - Taipei, 2015, 53(3), P. $0802 / 1-12$.

[28] Calogero F. and Degasperis A. Comparison between the exact and Hartree solutions of a one-dimensional many-body problem. Phys. Rev. A, 1975, 11(1), P. 265-269.

[29] Band R., Berkolaiko G., Raz H. et al. The Number of Nodal Domains on Quantum Graphs as a Stability Index of Graph Partitions. Commun. Math. Phys., 2012, 311, P. 815-838.

[30] Band R., Oren I., Smilansky U. Nodal domains on graphs - how to count them and why? In: Analysis on graphs and its applications. Proc. Sympos. Pure Math., 2008, 77, P. 5-27.

[31] Helffer B., Sundqvist M.P. On nodal domains in Euclidean balls. Proc. Amer. Math. Soc., 2016, 144(11), P. 4777-4791. 